\title{
Improving Water Supply System Components Visualization into GPR Images
}

\author{
By David Ayala-Cabrera* \\ Silvia J. Ocana-Levario ${ }^{\dagger}$ \\ Joaquin Izquierdo \\ Rafael Perez-Garcia ${ }^{+}$
}

This work focuses on the use of easy-to-apply procedures that allow rapid visualization of components of water supply systems (WSSs) by non-highly qualified personnel. We use a methodology that does not alter the conditions and characteristics of the environment (nondestructive methods), specifically the study of images obtained with ground penetrating radar (GPR). The study is based on the analysis and interpretation of the wave amplitude, then applying a series of image corrections, so that the display and handling of data is improved. The results are promising as a subjective and repeatable methodology to visualize buried pipes efficiently. The goal is to generate know-how to be able to train intelligent systems for the characterization of components of WSSs.

\section{Introduction}

Currently, technology inclusion into civil engineering fields, such as water supply systems (WSSs), is a challenge for researchers and utility managers. Data obtained after the incorporation of certain technologies should improve decision-making processes about the actions to propose for the assets of those systems. In this sense, non-destructive methods have shown to be interesting techniques that support network components assessment without affecting the surrounding environmental conditions. However, the huge size and the difficulty of interpretation of the obtained information, together with continuous network growth (population increase), maintenance activities, etc., greatly impair the power of these tools. These aspects constitute the main reasons for some technologies not to have been included efficiently in WSS management so far. In this paper, ground penetrating radar (GPR) is used as a non-destructive method to assess the components of WSSs. GPR selection is here considered due to its characteristics as a non-destructive method, which allows to discover layouts and features in both metallic and non-metallic pipes.

\footnotetext{
*PhD Student, FluIng-IMM, Polytechnic University of Valencia, Spain.

${ }^{\dagger}$ PhD Student, FluIng-IMM, Polytechnic University of Valencia, Spain.

†Professor, FluIng-IMM, Polytechnic University of Valencia, Spain.

${ }^{+}$Professor, Polytechnic University of Valencia, Spain.
} 
GPR has also important applications in discovering other WSS characteristics such as leaks [Crocco et al., 2010]. The aim is the detection of various pipe materials (such as plastic and metallic, among others), and the identification of some important characteristics (e.g. water leakage).

Several authors have addressed GPR image preprocessing, trying to improve visualization of buried objects. This process is performed in order to clean the noise of GPR images. This noise can be originated by the process itself, or by external signals embedded in the underground material gathered on the signal path. The aim is better visualization of the contrasting images and observing features of the objects under the ground. This is the way in which image preprocessing in WSSs is performed by the application of procedures adapted from other methodologies. This is the case of the application of background removal and migration, used by [Olhoeft, 2000] for cleaning GPR images to find metallic pipes. Works such as the one by [Simi et al., 2008] use the Hough transform in pattern recognition of the hyperbolas formed in GPR images. There also are works regarding leakage as the one by [Tavera, 2008], in which Hilbert and Fourier transforms are employed. Other works, based on intelligent systems for automatic pipe detection by GPR images use neural networks [Al-Nuaimy et al., 2000], support vector machines [Pasolli et al., 2008], fuzzy logic [Ciu et al., 2010], or multi-agent methodologies [AyalaCabrera et al., 2011], among others.

This work focuses on improving the visualization of WSS components into images captured using GPR. This seeks to encourage direct identification of these components in GPR images by personnel non-highly skillful in the interpretation of data obtained from non-destructive techniques, and to promote the design of automatic detection algorithms. This last aspect enables, for example, the integration of augmented reality techniques into the systems, which will support decision-making activities (regarding maintenance and rehabilitation, among others). This will represent a step forward in pursuance of the concept of the so-called Smart City approach, which includes autonomous management of WSSs, thus jointly benefiting utilities and citizens.

\section{Proposed Methodology}

In previous work [Ayala-Cabrera et al., 2014.a], we have demonstrated the feasibility of obtaining various forms from GPR images beyond the classical hyperbolas. These forms reflect the response of the waves emitted by different buried objects. They also contain information of the electromagnetic characteristics of the observed object, and its relationship to the electromagnetic properties of the surrounding environment. Additionally, we have observed that these forms can be fed into various processes, such as augmented reality [Ayala-Cabrera et al., 2013.c, 2013.d]. Such visualizations are intended to assist in water supply systems, both in operational and decisionmaking activities. 
Also, in other studies, we have shown that it is feasible to obtain other forms of events from GPR images. This is the case of water leaks [AyalaCabrera et al., 2013.b], and even the detailed study of their evolution [AyalaCabrera et al., 2014.b]. This aspect is crucial for leak detection in WSS and for understanding the phenomenon.

In this work, we intend to move forward towards an improved visualization of WSS components from GPR images. To this end, as a starting point, we propose using the timeline matrices obtained by the algorithm presented in [Ayala-Cabrera et al., 2013.a]. In this paper we propose a suitable correction of those timelines. With this correction, picture profiles, which are understandable by non-highly qualified personnel in the interpretation of images obtained with GPR, are obtained. The components of the proposed system are presented below.

\section{Brief Introduction of the Pre-Processing Algorithm}

The analysis carried out in this section is based on intensity differences demarcated by the wave amplitude generated in the images after the passage of the signal through various subsurface strata. The analysis we present in this section is based on time characteristics. In this analysis, the peaks (both maxima and minima) of the waves generated are extracted. The trend of the path of each trace and the average value of peak-to-peak time are studied. The basic principle assumes that the field is homogeneous, and thus, there should be a clear correspondence between the various peaks obtained. However, it should be noted that although the material is homogeneous, in practice, the measured values are different, even though very close together. Consequently, very different values demarcate anomalies in the image. These peaks are first extracted and numbered according to their occurrence in the trace. They are then placed in their respective positions in distance, and finally, the last value is used to fill an array of a certain size.

The pre-processing of GPR images used in this document was proposed in [Ayala-Cabrera et al., 2013.a] and was termed an agent race. The algorithm is developed in MatLab, is based on game theory and uses the multi-agent paradigm [Shoham et al., 2009]. Agent racing provides an interpretation and a grouping method for data from GPR radargrams. In this pre-process, we reduce the amount of data in the initial radargram, while preserving its initial properties and the most relevant data, so that its ability to identify buried objects through suitable visualizations is preserved. The multi-agent approach makes analysis much quicker. The input to this algorithm is the resulting radargram of the GPR prospection, which consists of an $m \times n$-sized matrix. The $n$-traces, of length $m$, that are generated are used in this work as parallel tracks for the $n$-agents to run. The race is an endurance test for the agents with a prize consisting in advancing one position depending on the effort made. Efforts are based on wave amplitude values in each column of the matrix (radargram). The race lasts a total time of $t=m$. The movements of an agent in $t$ are conditioned by the changing trend of the wave amplitude of the trace the agent runs. The race ends when time $t$ has elapsed. The winner is the agent who manages to 
obtain more movements (higher reward) during this time. The output of this process is a matrix, that we call $B$, of size $m 1 \times n$, where $m 1=$ the maximum number of movements. The columns of this matrix describe the movement of the agents in relation to the competition. In this work, the various movements developed by the agents are termed timelines. Figure 1 summarizes the GPR image preprocessing principles of the proposed system.

Figure 1. Principles of the GPR Image Pre-Processing Proposed. (a) Raw Image, (b) Trace, (c) Trace in Absolute Wave Amplitude Value, (d) Time Selection, and (e) Timeline Matrix; B Matrix. Source [Ayala-Cabrera et al., 2013.b]

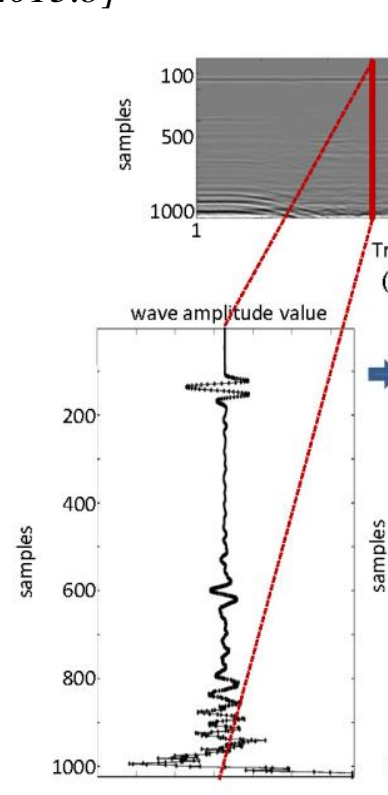

(b)

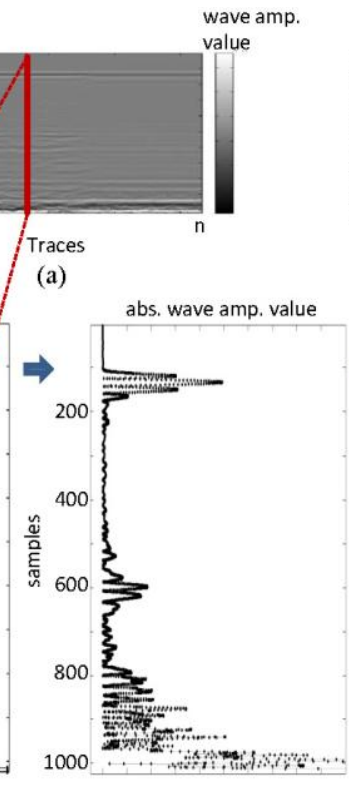

(c)

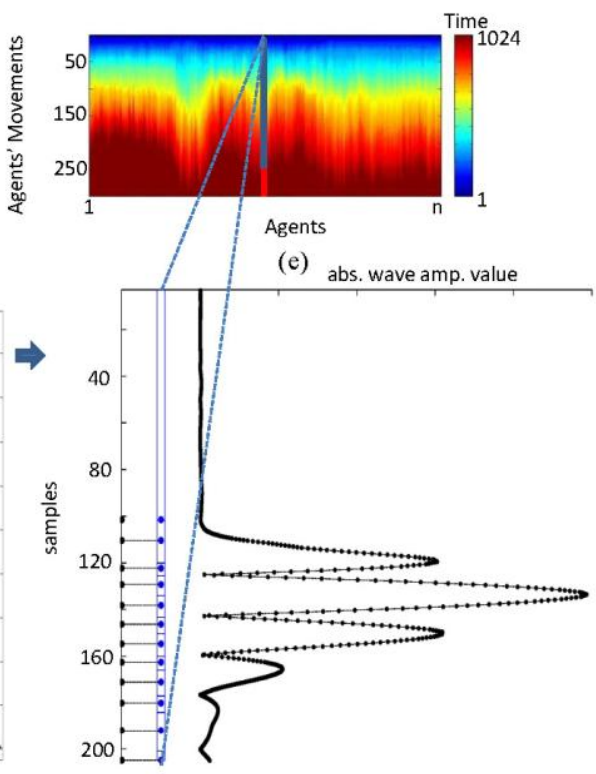

(d)

Timeline Corrections and Regrouping of Interest Objects

Let's consider $B$ the matrix with the timelines as columns. Since $B$ is an $m 1 \times n$ matrix, its elements are of the form $b_{i, j}$ with $I=\{1,2, \ldots, m 1\}$ and $j=$ $\{1,2, \ldots, n\}$. The additional process proposed in this paper is described next. It is intended to obtain a reorganization of this matrix that allows clearer and more interpretable visualization of the images. The first part of this process suitably corrects the timelines obtained. The initial expression is:

$$
d t_{i, j}=\left(\left(a b s\left(b_{i+1, j}-b_{i, j}\right)\right)>t h r\right) \cdot b_{i+1, j}
$$

where thr is a threshold value determined by the user, which will serve to bring together the timelines. With this process, consecutive points at a distance lower than the elected $t h r$ are eliminated.

In the next step time non-zero values are taken up on their columns, thus letting the zero values drop. The pseudo code for this approach is presented in Table 1. 
Table 1. Pseudo Code - Time Correction in the Timeline Matrix

$$
\begin{aligned}
& \text { for } i i=1: m 1-1 \\
& \quad \text { If } d t_{i i, j}>0 \text { or } i i=1 \\
& d t_{i i, j}=d t_{i i, j} \\
& \text { else }\left[\begin{array}{c}
d t_{i i, j} \\
d t_{i i+1, j} \\
\vdots \\
d t_{m 1-1, j} \\
d t_{m 1, j}
\end{array}\right]=\left[\begin{array}{c}
d t_{i i+1, j} \\
d t_{i i+2, j} \\
\vdots \\
d t_{m 1, j} \\
0
\end{array}\right] \\
& \text { end } \\
& \text { end }
\end{aligned}
$$

Next, bottom timelines consisting just of zeroes are eliminated (see Table 2).

Table 2. Pseudo Code to Eliminate Void Rows

$$
\begin{aligned}
& \text { ii }=0 \\
& \text { while ind }=0 \\
& \quad \text { do } \sum_{j=1}^{n} d t_{i i, j} \\
& \quad \text { if } \text { ind }^{\prime}>0 \\
& \quad\left[d t t_{i i, 1}, d t t_{i i, 2}, \ldots, d t t_{i i, n}\right]=\left[d t_{i i, 1}, d t_{i i, 2}, \ldots, d t_{i i, n}\right] \\
& \quad i i=i i+1 \\
& \text { end } \\
& \text { end }
\end{aligned}
$$

On each timeline, times obtained in this new matrix are ordered decreasingly. These timelines are then normalized. As a result, entries of the new matrix range between 0 and 1 .

In the next step, three groups of elements are defined within the matrix through the application of expression:

$d t t=\left((\operatorname{round}((d t t+1) \odot 2)) \odot \frac{1}{2}\right)$,

where, $\odot$ is the Hadammard product. Now the elements of the matrix belong to the set $\{1.0,1.5,2.0\}$. In this work we are interested in elements equal to 2.0. Figure 2 shows an example of the above described process. 
Figure 2. Example of Proposed Analysis: (a) Raw GPR Image, (b) PreProcessed Image - Timeline Image, (c) Ordered Timelines Image, Using thr = 20, (d) Classification Into 3 Groups, (e) Extraction of Profiles for Group 2.0, and Demarcation of Areas Containing More Than 200 Pixels

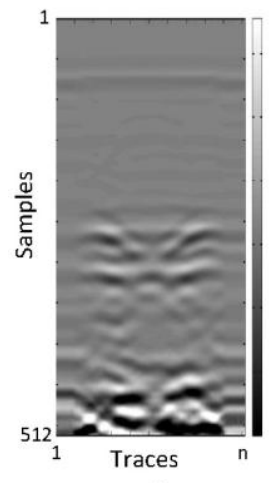

a)

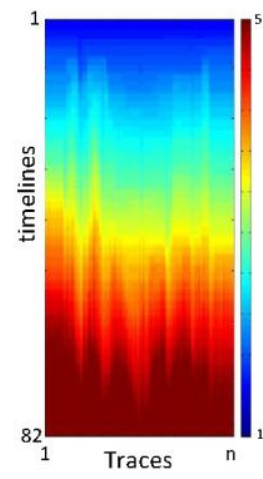

b)

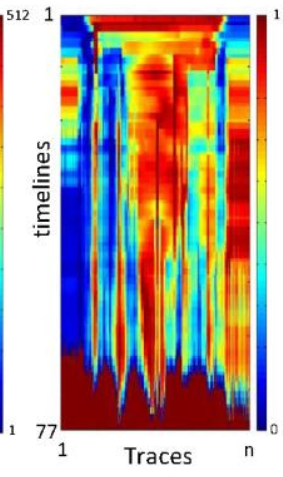

c)

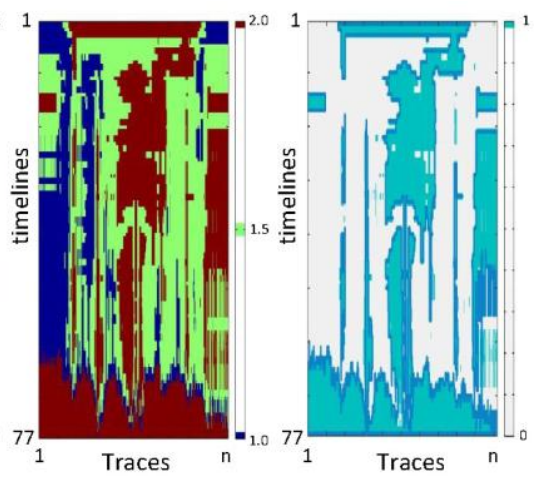

d)

e)

The final part of the proposed process (see Figure 2, e) is the removal of clusters with small amounts of pixels inside. It should be noted that, although this last part of the process (group removal) was performed manually, it can be automated, with the evaluation of shape parameters (area, perimeter, eccentricity, etc.) for the groups obtained. It should be noted that the shape parameter that prevailed in this document was the area of the obtained groups.

\section{Case Study - Analysis of Results}

This section discusses the implementation of the proposed system in the previous section to improve the visualization of components of WSSs from GPR images. The case study corresponds to GPR images of pipes of four pipe materials commonly used in WSSs. The pipe materials used in this work are polyvinyl chloride (PVC), polyethylene, asbestos-cement, and cast iron. The nominal diameter of the pipes used is $0.10 \mathrm{~m}$. GPR images were obtained by burying the pipes in dry soil in a test tank. Additionally, it should be mentioned that the asbestos-cement pipe had a lime incrustation inside.

Two trials for each pipe, plus two additional measurements without pipe, were undertaken. These last two measurements were performed with the aim of making visible the difference between the images with and without pipe. Two types of antenna with center frequencies of $1500 \mathrm{MHz}$ and $900 \mathrm{MHz}$, one for testing and another for configuration, were used. The parameters of the equipment correspond to 120 traces/s, 512 samples/trace and 20ns/512samples.

The aim of the proposed system is to obtain profiles similar to the ones of the schemes. The performance of the proposed system in the conducted tests is presented in Figure 3. 
Figure 3. (a-e) Schematic Configuration-Image Objective, $(f-j)$ Results Using the GPR 1500MHz Antenna, (k-o) Results Using the 900MHz Antenna. ( $a, f, k)$ Reference Test - without Pipe, $(b, g, l)$ Test with PVC Pipe, $(c, h, m)$ Test with Polyethylene Pipe, $(d, i, n)$ Test with Asbestos-Cement Pipe and Internal Incrustation, $(e, j, o)$ Test for Cast Iron Pipe

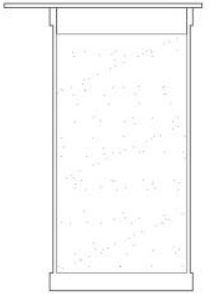

a)

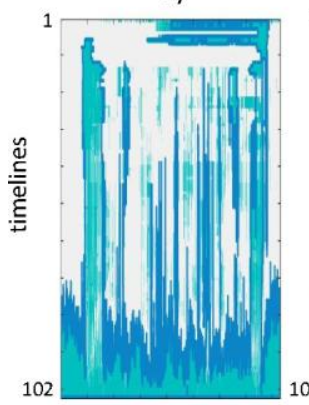

f)

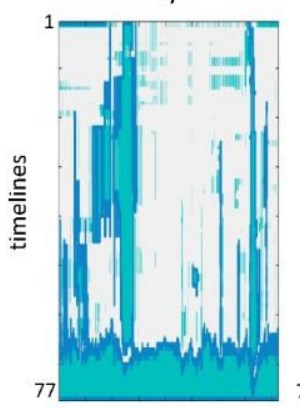

k)

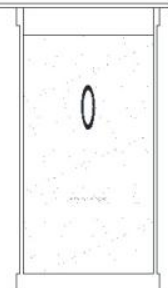

b)

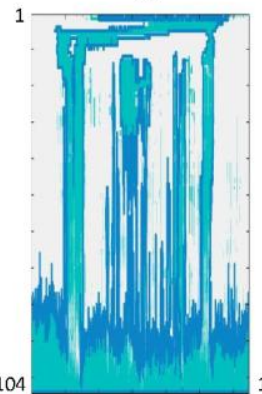

g)

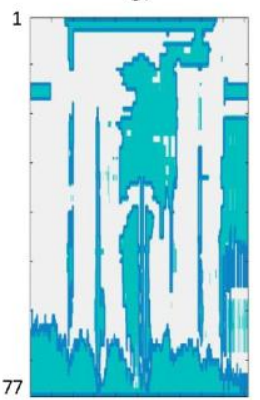

l)

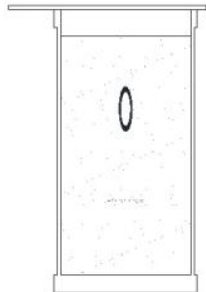

c)

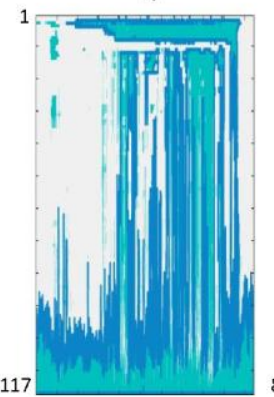

h)

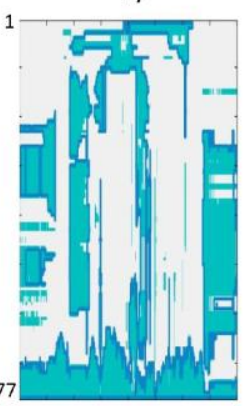

m)

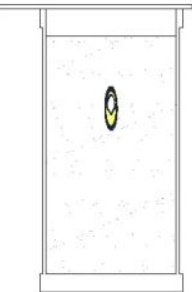

d)

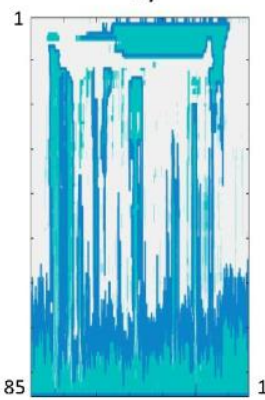

i)

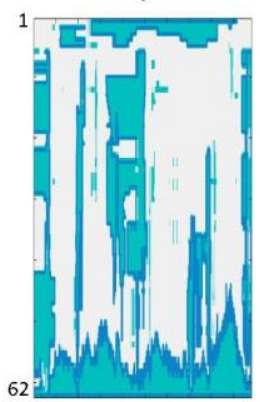

n)

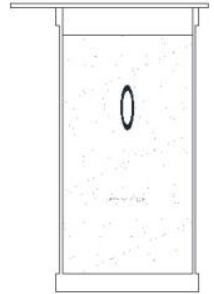

e)

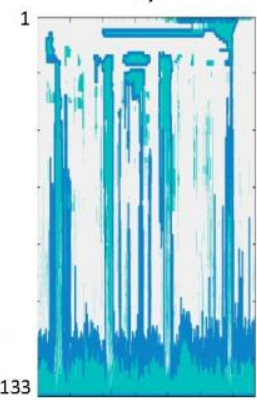

j)

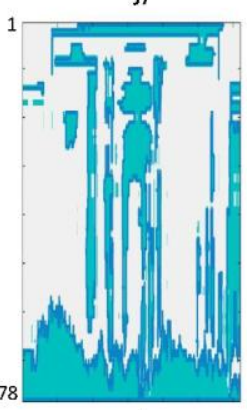

o)

In the tests obtained with the $1500 \mathrm{MHz}$ antenna (Figure 3, f to j) better detailed shapes are observed than those obtained in the images with the 900 $\mathrm{MHz}$ antenna (Figure 3, $\mathrm{k}$ to o). For both antenna configurations the anomaly added to the reference test, namely each of the pipes, can be clearly observed.

For the polyethylene pipe (Figure 3, h and $\mathrm{m}$ ), it can be seen how the shape formed can hardly be distinguished from the surrounding medium. This reduced contrast is the result of the pipe dielectric properties similar to those of the surrounding medium. This feature can also be observed in the case of the PVC pipe, although less so than for polyethylene. This underlines the difficulty in visualizing plastic materials in GPR images.

Moreover, it is clear how regarding metallic elements (Figure 3, j and o), the signals are more intense. Lines surrounding the pipe are also observed, what even reduces the visualization of the tank walls.

It can be seen thus, how visual elements are embedded into the environment. This clearly hinders their interpretation visually. 
While it is true that the profiles obtained are likely to be improved, so is that they are a good starting point to focus on aspects such as pipeline location and classification, and, in general, interpretation of GPR images.

\section{Conclusions}

This paper proposes correcting the timelines obtained by the algorithm proposed by [Ayala-Cabrera et al., 2013.a]. The objective is to improve the visualization of WSS components in GPR images. With this correction, the visualization of images of pipes buried in dry soil is improved. In general, after the application of the proposed system considerable improvement when working with visualization and consolidation of characteristics of the pipes in the GPR images is achieved. This helps show clearly defined lines, which, in turn, can be grouped so that the resulting images approach the proposed schematic configurations.

Finally, it should be noted that this is a simple process that does not depend on specialist skills, and is repeatable. The proposed system is efficient with computational resources (even in the problematical case of plastic pipes). In all the cases, even though the amount of information dealt with is reduced, reliability is preserved. Moreover, the proposed method offers the possibility of more detailed analysis, and this creates the possibility of better interpretations that could serve as a basis for intelligent training systems. This approach would help give WSS managers a more accurate vision of the systems they operate and, as a result, offer a better service to users.

\section{Acknowledgments}

Part of this work has been developed under the support of an FPI (Formación de Personal Investigador)-UPV (Universitat Politècnica de València) scholarship granted to the first author by the Programa de Ayudas de Investigación y Desarrollo (PAID) of the Universitat Politècnica de València.

\section{References}

Al-Nuaimy, W., Huang, Y., Nakhka, S. H. M., Fang, M. T. C., Nguyen, V. T., and Eriksen, A. 2000. Automatic detection of buried utilities and solid objects with GPR using neural networks and pattern recognition. J. Appl. Geophys; 43, 2-4 (March. 2000), 157-165. DOI= http://dx.doi.org/10.1016/S0926-9851(99)00055$\underline{5}$.

Ayala-Cabrera, D., Izquierdo, J., Ocaña-Levario, S. J., and Pérez-García, R. 2014.a. 3D model construction of water supply pipes based on GPR images. In Proceedings of the 7th International Congress on Environmental Modelling and Software (San Diego, California, USA, June 15-19, 2014). URL: http://www.iem ss.org/society/index.php/iemss-2014-proceedings. 
Ayala-Cabrera, D., Campbell, E., Carreño-Alvarado, E. P., Izquierdo, J., and PérezGarcía, R. 2014.b. Water leakage evolution based on GPR interpretations. In Proceedings of the Water Distribution System Analysis 2014 (Bari, Italy, 2014).

Ayala-Cabrera, D., Izquierdo, J., Montalvo, I., and Pérez-García, R. 2013.a. Water supply system component evaluation from GPR radargrams using a multi-agent approach. Math. Comput. Model. 57, 7-8 (April. 2013), 1927-1932. DOI= http:// dx.doi.org/10.1016/j.mcm.2011.12.034.

Ayala-Cabrera, D., Herrera, M., Izquierdo, J., Ocaña-Levario, S. J., and Pérez-García, R. 2013.b. GPR-based water leak models in water distribution systems. Sensors 13, 12 (Nov. 2013), 15912-15936. DOI= http://dx.doi.org/10.3390/s131215912.

Ayala-Cabrera, D., Herrera, M., Izquierdo, J., Pérez-García, R., and Ocaña-Levario, S. J. 2013.c. Dynamic management of water supply systems: A tool to build scenarios by merging GPR surveys and augmented reality. Water Utility Journal 6, (July-Dec. 2013), 3-8.

Ayala-Cabrera, D., Ocaña-Levario, S. J., Pérez-García, R., Izquierdo, J., and Herrera, M. 2013.d. GPR y realidad aumentada: hacia la visualización automática de tuberías en SAA. In Proceedings of Seminario Euro Latinoamericano de Sistemas de Ingeniería 2013 (La Victoria, Venezuela, November 12-15, 2013).

Ayala-Cabrera, D., Herrera, M., Izquierdo, J., and Pérez-García, R. 2011. Location of buried plastic pipes using multi-agent support based on GPR images. J. Appl. Geophys. 75, 4 (Dec. 2011), 679-686. DOI= http://dx.doi.org/10.1016/ j.jappgeo. 2011.09.024.

Ciu, Y., Wang, L., and Xiao, J. 2010. Automatic feature recognition for GPR image processing. World Academy of Science, Engineering and Technology 61, 176179.

Crocco, L., Soldovieri, F., Millington, T., and Cassidy, N. J. 2010. Bistatic tomographic GPR imaging for incipient pipeline leakage evaluation. Prog. Electromagn. Res. 101, 307-321. DOI= http://dx.doi.org/10.2528/PIER091222 06.

Olhoeft, G. R. 2000. Maximizing the information return from Ground penetrating radar. J. Appl. Geophys. 43, 2-4 (March 2000), 175-187. DOI= http://dx.doi.org/ 10.1016/S0926-9851(99)00057-9.

Pasolli, E., Melgani, F., and Donelli, M. 2008. Automatic detection and classification of buried objects in GPR images using genetic algorithms and support vector machines. In Proceedings of the Geoscience and Remote Sensing Symposium, IGARSS, 2008 (Boston, MA, USA, July 7-11, 2008). 525-528. DOI= http://dx. doi.org/10.1109/IGARSS.2008.4779044.

Shoham, K., and Leyton-Brown, K. 2009. Multiagent Systems: Algorithmic, GameTheoretic and Logical Foundations. Cambridge University Press. Cambridge, MA, USA.

Simi, A., Bracciali, S., and Manacorda, G., 2008. Hough transform based automatic pipe detection for array GPR: algorithm development and on-site test. In Proceedings of the Radar Conference, 2008, RADAR '08. IEEE (Rome, Italy, May 26-30, 2008). 1-6. DOI= http://dx.doi.org/10.1109/RADAR.2008.4720763.

Tavera, M. 2008. Aplicación de Georadar para la Mejora del Rendimiento de una Red Hidrica. Master Thesis, Polytechnic University of Valencia, Valencia, Spain. 
